\title{
14. ICHTHYOLITHS AT SITE 464 IN THE NORTHWEST PACIFIC, DEEP SEA DRILLING PROJECT LEG $62^{1}$
}

\author{
P. S. Doyle and W. R. Riedel, Scripps Institution of Oceanography, La Jolla, California
}

\section{INTRODUCTION}

At Site 464 (Fig. 1), a 308-meter-deep hole was drilled on the Hess Rise $\left(39^{\circ} 51.64^{\prime} \mathrm{N}, 173^{\circ} 53.33^{\prime} \mathrm{E}\right.$; water depth $4637 \mathrm{~m}$ ). The upper strata consist of siliceous clay and oozes of Pleistocene, Pliocene, and possibly latest Miocene ages $(36 \mathrm{~m})$ and the lower strata are $\mathrm{Al}$ bian chert, chalk, and marlstone $(219 \mathrm{~m})$. The middle part of the stratigraphic section is pelagic brown clay ( 53 $\mathrm{m}$ ) which is essentially barren of microfossils except for ichthyoliths. At the base of this middle unit in Core 10 is a recrystallized Cretaceous radiolarian assemblage and an impoverished late Miocene nannofossil assemblage. Fifteen samples from Cores 6 through 10 in the middle unit and three samples from the overlying siliceous clay were examined for ichthyoliths.

The method of preparation and the system of discrimination and nomenclature used is that of Doyle et al. (1974), as modified in subsequent papers (Dunsworth et al., 1975; Ramsey et al., 1976; Edgerton et al., 1977; Doyle et al., 1978; Doyle and Riedel, in press a).

All ichthyoliths previously described have been looked for in the Site 464 samples (except for Triangle complex transverse line, which appears erratically throughout the Late Cretaceous and Cenozoic, and Triangle inline halfway, which is not yet very useful stratigraphically), and the numbers of specimens of each subtype found are recorded in Table 1. In the table, the subtypes are arranged in the order of their earliest occurrences in samples having age assignments based on other microfossils. In the top part of the table is a summary of the published records of the subtypes in such dated samples (Doyle et al., 1974; Dunsworth et al., 1975; Ramsey et al., 1976; Doyle et al., 1977; Doyle et al., 1978; Doyle and Riedel, in press a,b,c). The numbers of specimens given for Triangle smooth surface and Spiky palmate are from a manuscript in preparation on Cretaceous-Paleogene ichthyoliths. The numbers of hundreds of triangular ichthyoliths found in these samples, for each time period, are shown in the far right column. Figure 2 summarizes the results from the table.

\section{RESULTS}

Comparison of the order of occurrences of the subtypes in the Site 464 sequence with that in the dated

\footnotetext{
${ }^{1}$ Initial Reports of the Deep Sea Drilling Project, Volume 62.
}

samples suggests the following age assignments: Cores 4 and 5 are middle Miocene or younger; Core 6, Sections 1 and 3, might be late Eocene, Oligocene, or early Miocene; Core 6, Section 5, is early Eocene; Cores 7 and 8 are Paleocene; and Cores 9 and 10 are late Cretaceous.

Placement of the Cretaceous/Paleocene boundary between Cores 8 and 9 is open to question. The tabulated early Paleocene occurrences of subtypes Blunt triangle dendritic inline, Striated blunt triangle, Wide triangle projection, Triangle square inline, Pointed and skirted, Prominent polygon, Centrally striated triangle, and Triangle long inline are based mainly on three samples from Cores 10 and 11 at Site 361 (Doyle et al., 1978, table 1). Those two cores were assigned to the early Paleocene on the basis of benthic foraminifers (ProtoDecima and Bolli, 1978). However, at other locations (Doyle and Riedel, in press b, table 3), most of these subtypes are confined to the Cretaceous and do not occur in the Paleocene.

The other problematic part of the Site 464 section is in Core 6, where Sections 1 and 3 cannot be dated more precisely than late Eocene to early Miocene. These samples contain Rounded apex triangle and Flexed triangle $102-112$, both having one occurrence each in the late Eocene, but with the coherent range of each beginning in the late Oligocene (see top of Table 1). On the other hand, none of the subtypes with first occurrences in late Oligocene appears in Sections 6-1 and 6-3. It seems clear that this interval is no younger than early Miocene, because of the presence of Flexed triangle shallow inbase $\geq 120$, Curved triangle inline constricted, Triangle short wing, Triangle pointed margin ends, and Triangle medium wing.

Figure 2 shows a peak in ichthyolith abundances at the top of Core 6, which seems to correlate with a peak observed in the late Eocene-Oligocene at two other North Pacific locations (DSDP Site 436 and Giant Piston Core-3; Doyle and Riedel, in press a, figs. 1,2). There is, however, no equivalent of the late Paleocene or early Eocene peak observed at those two locations, possibly because our samples from Site 464 are too widely spaced or because part of the sediment column is missing.

\section{ACKNOWLEDGMENTS}

This research was supported partly by NSF Grant OCE76-80884, and partly by the University of California. The task of typing was competently performed by Mary Hanger. David Bukry (U.S. Geological Survey) and Ansis Kaneps (Scripps Institution of Oceanography) reviewed the manuscript. 
Table 1. Distribution of ichthyolith subtypes in Cores 464-4 through 464-10.

\begin{tabular}{|c|c|c|c|c|c|c|c|c|c|c|c|c|c|c|c|c|c|c|c|c|c|c|c|c|c|c|}
\hline \multirow{8}{*}{ 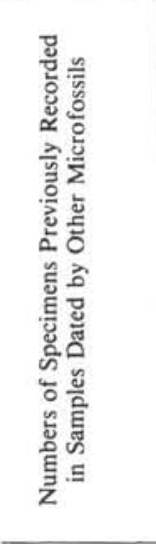 } & $\begin{array}{l}\text { Quaternary } \\
\text { Pliocene }\end{array}$ & & & & & & & & & & & & & & & & & & \multirow{2}{*}{\multicolumn{2}{|c|}{1}} & \multirow{2}{*}{$\frac{1}{1}$} & \multirow[t]{2}{*}{1} & \multicolumn{3}{|c|}{$\begin{array}{ll}1 & 3 \\
1 & 3 \\
\end{array}$} & \\
\hline & & & & & & & & & & & & & & & & & & & & & & & 1 & 15 & & \\
\hline & $\begin{array}{cc}\text { Miocene } & 1 \\
\mathrm{~m} \\
\mathrm{e}\end{array}$ & & & & 5 & $\begin{array}{l}1 \\
1 \\
1\end{array}$ & & & & & & & & & & & & & & & $\begin{array}{l}1 \\
1\end{array}$ & 1 & $\begin{array}{l}2 \\
1 \\
3\end{array}$ & $\begin{array}{r}5 \\
6 \\
32\end{array}$ & 1 & 1 \\
\hline & & & & & & 2 & & & & & & & & & & & & & & & & & 1 & 28 & 2 & 2 \\
\hline & $\begin{array}{ll}\text { Oligocene } & 1 \\
& \mathrm{e}\end{array}$ & & 1 & 1 & & 4 & & & & & & & & & & & 1 & 1 & 3 & & 1 & 1 & 13 & $\begin{array}{l}65 \\
15 \\
\end{array}$ & $\begin{array}{r}2 \\
19 \\
\end{array}$ & $\begin{array}{l}4 \\
8 \\
\end{array}$ \\
\hline & $\begin{array}{cc} & 1 \\
\text { Eocene } & \mathrm{m} \\
& \mathrm{e} \\
\end{array}$ & 3 & 2 & 2 & 2 & $\begin{array}{r}1 \\
6 \\
10 \\
\end{array}$ & & & & & & & & & & 1 & 6 & 1 & $\begin{array}{l}3 \\
2 \\
4 \\
\end{array}$ & 1 & $\begin{array}{r}1 \\
18 \\
16 \\
\end{array}$ & 4 & $\begin{array}{r}6 \\
8 \\
29 \\
\end{array}$ & $\begin{array}{r}41 \\
108 \\
128 \\
\end{array}$ & $\begin{array}{r}5 \\
26 \\
17 \\
\end{array}$ & $\begin{array}{r}1 \\
18 \\
21 \\
\end{array}$ \\
\hline & $\begin{array}{ll}\text { Paleocene } & 1 \\
\mathrm{e}\end{array}$ & $\begin{array}{rr}3 \\
1 \quad 16 \\
\end{array}$ & 9 & $\begin{array}{l}3 \\
1\end{array}$ & 1 & 1 & 2 & 2 & 3 & 4 & 84 & 13 & 50 & 7 & 6 & $\begin{array}{l}2 \\
1\end{array}$ & $\begin{array}{ll}6 & 1 \\
1 & \\
\end{array}$ & $\begin{array}{r}16 \\
2\end{array}$ & 4 & $\begin{array}{l}5 \\
9\end{array}$ & $\begin{array}{l}5 \\
7 \\
\end{array}$ & $\begin{array}{r}9 \\
45 \\
\end{array}$ & 8 & 15 & $\begin{array}{r}20 \\
3 \\
\end{array}$ & $\begin{array}{l}58 \\
13 \\
\end{array}$ \\
\hline & $\begin{array}{ll}\text { Cretaceous } & \mathrm{I} \\
\mathrm{e}\end{array}$ & $\begin{array}{rr}9 & 5 \\
5 & 11\end{array}$ & $\begin{array}{r}14 \\
5\end{array}$ & $\begin{array}{r}30 \\
4\end{array}$ & $\begin{array}{l}6 \\
3\end{array}$ & $\begin{array}{r}15 \\
5\end{array}$ & $\begin{array}{r}17 \\
1\end{array}$ & 25 & 22 & 16 & 14 & 4 & 59 & 18 & 4 & 25 & 1 & 1 & 21 & \begin{tabular}{l|l}
17 & 4
\end{tabular} & 41 & 40 & 27 & 2 & & \\
\hline $\begin{array}{c}\text { Ages } \\
\begin{array}{c}\text { Determined } \\
\text { by } \\
\text { Ichthyoliths }\end{array}\end{array}$ & $\begin{array}{c}\text { Sample } \\
\text { (core, section, } \\
\text { interval in } \mathrm{cm} \text { ) }\end{array}$ & 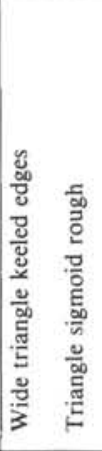 & 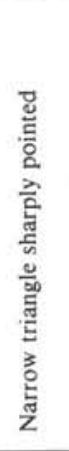 & 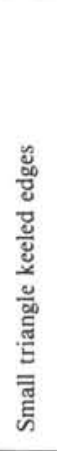 & 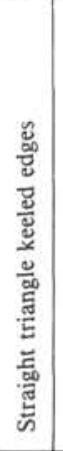 & 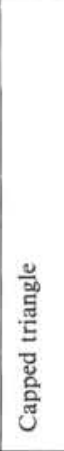 & 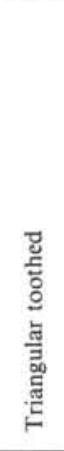 & 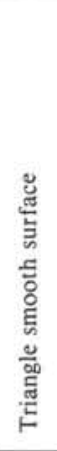 & 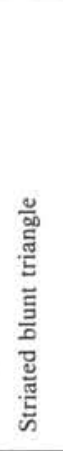 & 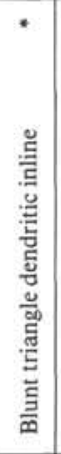 & 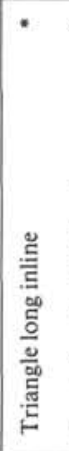 & 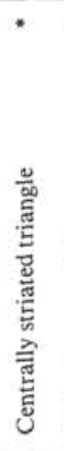 & 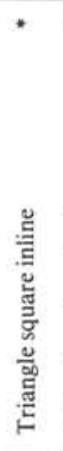 & 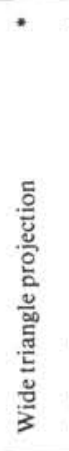 & 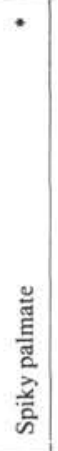 & 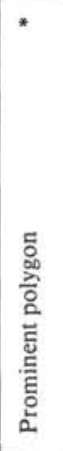 & 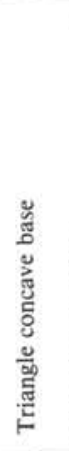 & 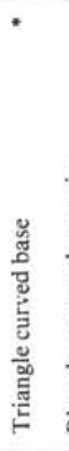 & 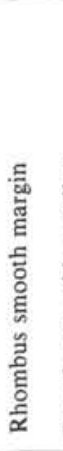 & 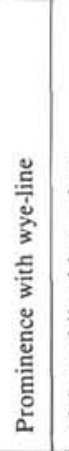 & 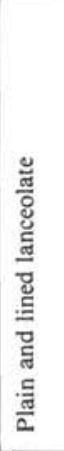 & 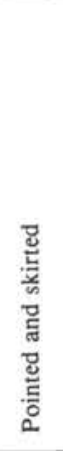 & 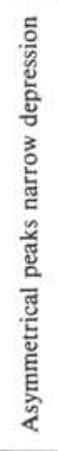 & 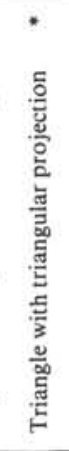 & 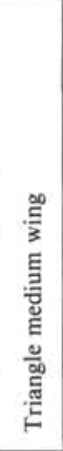 & 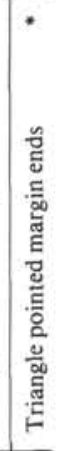 \\
\hline $\begin{array}{l}\text { mid. Miocene } \\
\text { or } \\
\text { younger }\end{array}$ & $\begin{array}{r}464-4-4,68-74 \\
5-2,72-78 \\
5-4,74-80 \\
\end{array}$ & & & & & & & & & & & & & & & & & & & & & & & 1 & & \\
\hline $\begin{array}{l}\text { e. Miocene/ } \\
\text { I. Eocene } \\
\text { e. Eocene }\end{array}$ & $\begin{array}{l}6-1,74-80 \\
6-3,74-80 \\
6-5,74-80 \\
\end{array}$ & 1 & & & & & & & & & & & & & & & $\begin{array}{c}\text { aff. } \\
2\end{array}$ & & $?$ & & $\begin{array}{l}2 \\
1\end{array}$ & 1 & 1 & $\begin{array}{r}17 \\
5 \\
11\end{array}$ & $\begin{array}{l}2 \\
3 \\
5 \\
\end{array}$ & $\begin{array}{r}3 \\
10 \\
\end{array}$ \\
\hline Paleocene & $\begin{array}{l}7-1,70-77 \\
7-3,70-77 \\
7-5,64-70 \\
8-1,64-70 \\
8-2,70-77 \\
\end{array}$ & & & & & & & & & & & & & & $?$ & & aff. & aff. & & & & & & $\begin{array}{l}3 \\
? \\
1 \\
1\end{array}$ & 1 & $\begin{array}{l}12 \\
10 \\
3 \\
2\end{array}$ \\
\hline $\begin{array}{c}\text { late } \\
\text { Cretaceous }\end{array}$ & $\begin{array}{l}9-1,70-77 \\
9-4,120-127 \\
9-6,70-77 \\
10-1,40-47 \\
10-2,40-47 \\
10-3,40-47 \\
10-4,40-47\end{array}$ & 1 & 1 & $\begin{array}{l}1 \\
1\end{array}$ & aff. & $\begin{array}{l}1 \\
1\end{array}$ & 1 & $\begin{array}{r}1 \\
1 \\
3 \\
12 \\
12 \\
7\end{array}$ & $\begin{array}{c}\text { aff. } \\
3 \\
2 \\
7 \\
1\end{array}$ & 1 & $\begin{array}{c}1 \\
\text { aff. } \\
?\end{array}$ & 2 & $\begin{array}{l}2 \\
1 \\
2 \\
1 \\
1\end{array}$ & $\begin{array}{c}\text { aff. } \\
\text { aff. } \\
1 \\
1 \\
1\end{array}$ & $?$ & 1 & & & & 2 & 1 & 1 & $\begin{array}{l}1 \\
1 \\
3 \\
1 \\
5\end{array}$ & & & \\
\hline
\end{tabular}

Note: The numbers in the body of the table are the total numbers of specimens found. Question marks represent broken specimens in samples containing no complete specimens of the subtype. The upper part of the table summarizes the occurrences of ichthyolith subtypes in samples with age assignments based on other microfossils (see text). Subtypes which we consider most reliable stratigraphically are marked with an asterisk.

\section{REFERENCES}

Doyle, P. S., Dunsworth, M. J., and Riedel, W. R., 1977. Reworking of ichthyoliths in eastern tropical Pacific sediments. Deep-Sea Res., 24:181-198.

1978. Ichthyoliths from some southeast Atlantic sediments, DSDP Leg 40. In Bolli, H. M., Ryan, W. B. F., et al., Init. Repts. DSDP, Suppl. to Vols. 38, 39, 40, and 41: Washington (U.S. Govt. Printing Office), 743-759.
Doyle, P. S., Kennedy, G. G., and Riedel, W. R., 1974. Stratignathy. In Davies, T. A., Luyendyk, B. P., et al., Init. Repts. DSDP, 26: Washington (U.S. Govt. Printing Office), 825-905.

Doyle, P. S., and Riedel, W. R., in press a. Ichthyoliths from Site 436, northwest Pacific, Leg 56, Deep Sea Drilling Project. In Scientific Party, Init. Repts. DSDP, 56, 57, Pt. 2: Washington (U.S. Govt. Printing Office).

, in press b. Cretaceous to Neogene ichthyoliths in a giant piston core from the central North Pacific. Micropaleont. 
Table 1. (Continued).

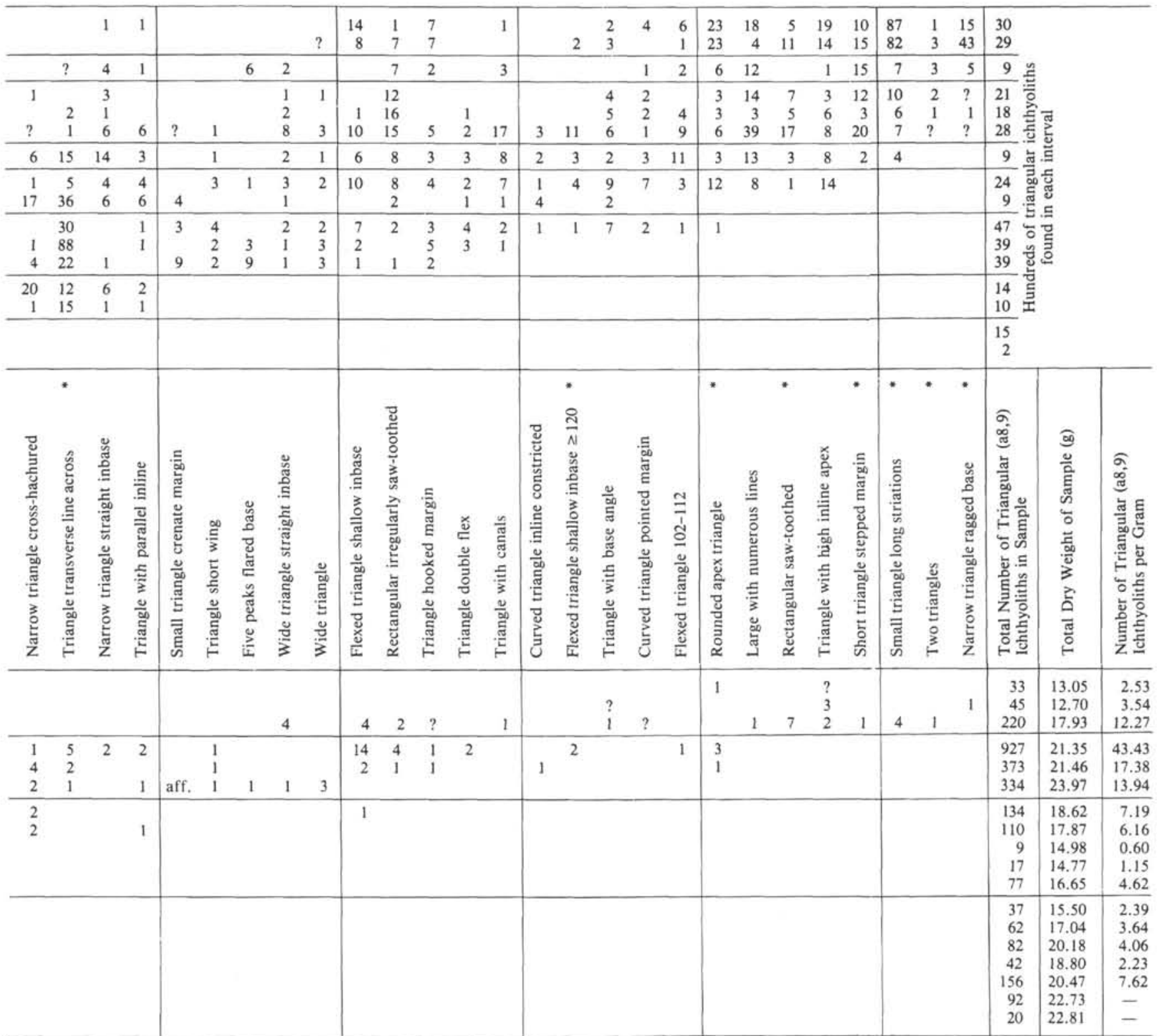

in press c. Ichthyoliths: present status of taxonomy and stratigraphy of microscopic fish skeletal debris. Scripps Inst. Oceanog. Ref. Ser., No. 79-16: Springfield, Va. (Natl. Tech. Info. Service).

Dunsworth, M. J., Doyle, P. S., and Riedel, W. R., 1975. Ichthyoliths from some NW Pacific sediments, DSDP Leg 32. In Larson, R. L., Moberly, R., et al., Init. Repts. DSDP, 32: Washington (U.S. Govt. Printing Office), 853-863.
Edgerton, C. C., Doyle, P. S., and Riedel, W. R., 1977. Ichthyolith age determination of otherwise unfossiliferous Deep Sea Drilling Project cores. Micropaleont., 23:194-205.

Proto-Decima, F., and Bolli, H. M., 1978. Southeast Atlantic DSDP Leg 40 Paleogene benthic foraminifers. In Bolli, H. M., Ryan, W. B. F., et al., Init. Repts. DSDP, 40: Washington (U.S. Govt. Printing Office), 783-810.

Ramsey, C. A., Doyle, P. S., and Riedel, W. R., 1976. Ichthyoliths in Late Mesozoic pelagic sediments, mainly from Italy. Micropaleont., 22:129-142. 
P. S. DOYLE, W. R. RIEDEL

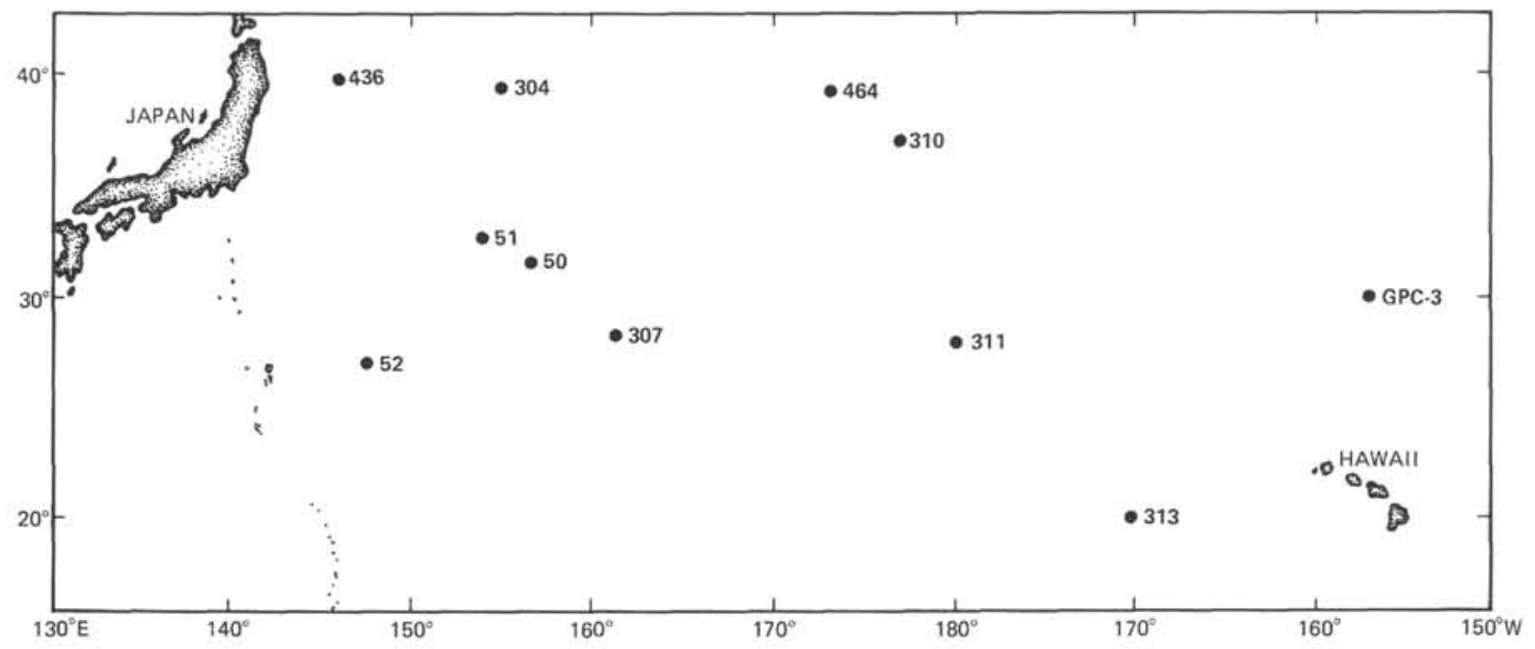

Figure 1. Location of Site 464 and other sites in the north Pacific from which ichthyoliths have been investigated (Dunsworth, et al., 1975; Edgerton, et al., 1977; Doyle and Riedel, in press a, b).

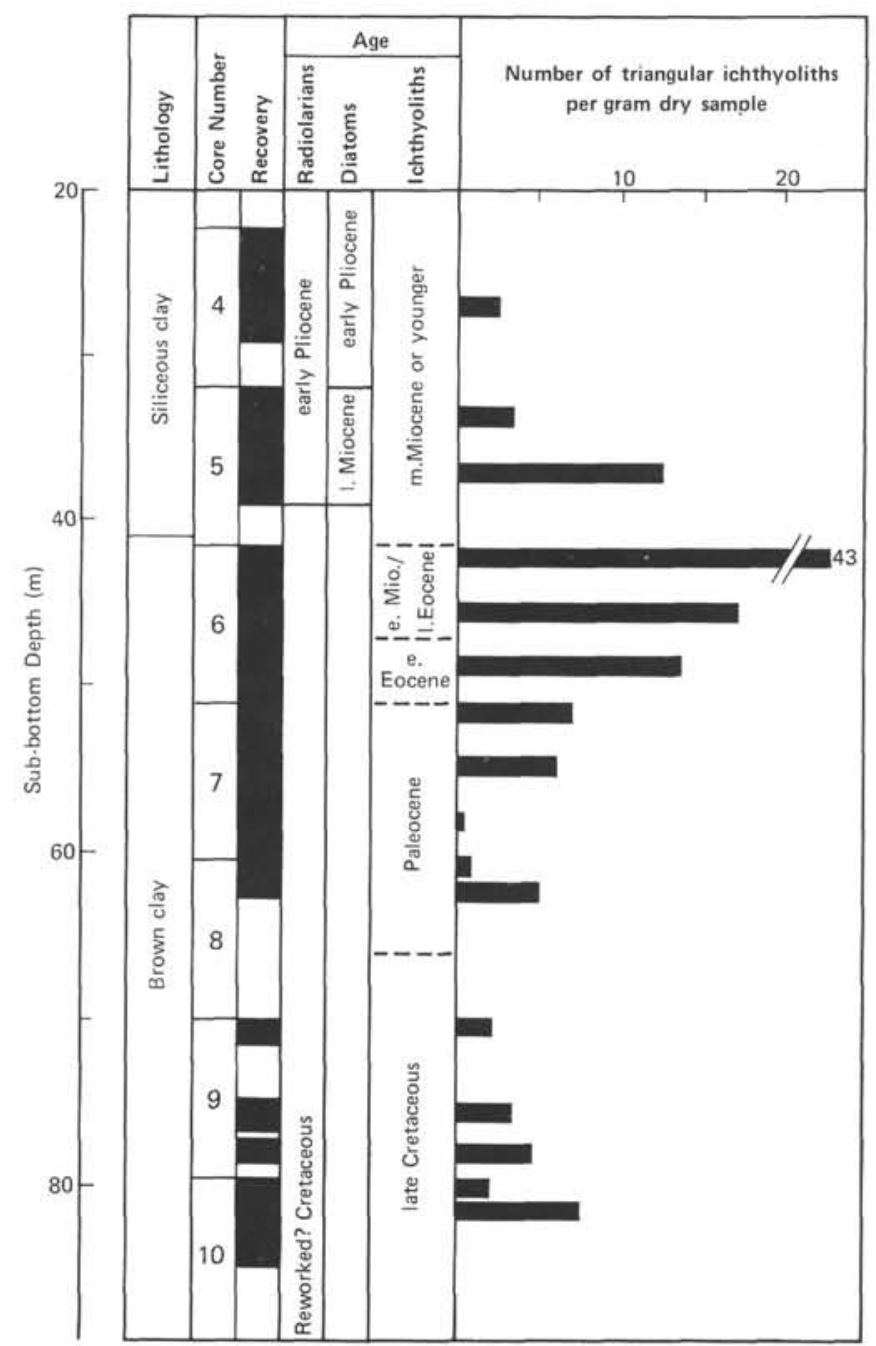

Figure 2. Diagrammatic summary of Site 464 , Cores 4 through 10. Horizontal bars on the right indicate the concentrations of triangular ichthyoliths in the sample studied. 